\title{
Espessamento Médio-Intimal na Origem da Artéria Subclávia Direita como Marcador Precoce de Risco Cardiovascular
}

Intima-Media Thickness in the Origin of Right Subclavian Artery as an Early Marker of Cardiovascular Risk

\author{
Carlos Alberto Engelhorn, Ana Luiza Engelhorn, Maria Fernanda Cassou, Cassiana Casagrande Zanoni, Carlos José \\ Gosalan, Emerson Ribas, Adriana Pacholok, Marcela de Fátima Koehler \\ Pontifícia Universidade Católica do Paraná e Angiolab, Curitiba, PR
}

Objetivo: O espessamento médio-intimal (EMI) na artéria carótida comum é considerado fator de risco cardiovascular e marcador de doença arterial coronariana precoce. O objetivo deste trabalho foi investigar a existência de correlação entre o EMI nas artérias carótidas e na origem da artéria subclávia direita, e avaliar o EMI na artéria subclávia como um marcador mais precoce para avaliação de risco cardiovascular.

Métodos: Cento e seis pacientes consecutivos, 52 homens e 54 mulheres, com média de idade de 51 anos, foram submetidos à avaliação das artérias carótidas e subclávia direita pela ultra-sonografia vascular com Doppler colorido. Para avaliar a associação entre EMI das artérias carótidas e subclávia direita calcularam-se o coeficiente de correlação de Pearson e o intervalo de 95\% de confiança para esse coeficiente. A qualidade da medida do EMI da artéria subclávia direita para diagnóstico de espessamento precoce, considerando-se o espessamento da carótida como padrão de referência ( $>0,8 \mathrm{~mm})$, foi descrita por valores de sensibilidade, especificidade, valor preditivo positivo, valor preditivo negativo e acurácia. Pontos de corte para o EMI da artéria subclávia foram sugeridos pela Curva ROC. Valores de $p \leq 0,05$ foram considerados estatisticamente significantes.

Resultados: Na associação entre 41 artérias carótidas sem EMI, 30 (73\%) artérias subclávias direitas apresentavam EMI > 0,8 $\mathrm{mm}$. O valor médio de EMI obtido na artéria carótida foi de $0,87 \mathrm{~mm}(\mathrm{DP}=0,23)$ e na artéria subclávia direita foi de 1,17 mm (DP = 0,46), com coeficiente de correlação de 0,31 (95\% IC: 0,12;0,47). A avaliação pela curva ROC demonstrou um valor de corte de $0,7 \mathrm{~mm}$ para EMI da artéria subclávia direita, tendo como padrão de referência o valor de corte de EMI da artéria carótida de 0,8 mm (sensibilidade 91\%, especificidade 27\%, VPP 66\%, VPN 65\% e acurácia 66\%).

Conclusão: Existe boa correlação entre o EMI das artérias carótidas e da artéria subclávia direita. O EMI pode ser detectado mais precocemente na artéria subclávia do que nas carótidas, com valor de corte de 0,7 mm. O EMI na origem da artéria subclávia direita pode ser considerado um marcador mais precoce para avaliação de risco cardiovascular.

Palavras-chave: Espessamento médio-intimal, arteriosclerose coronariana, infarto do miocárdio, diagnóstico precoce.

Objective: Common carotid artery intima-media thickness (IMT) is considered a factor of cardiovascular risk and an early marker of coronary artery disease. This study aimed to investigate the existence of a correlation between IMT in the carotid arteries and at the origin of the right subclavian artery, as well as to evaluate IMT in the subclavian artery as an earlier marker of cardiovascular risk.

Methods: One hundred and six consecutive patients, 52 males and 54 females, average age 51 years, underwent color Doppler ultrasonography to evaluate carotid and right subclavian arteries. The relationship between carotid IMT and right subclavian IMT was assessed using the Pearson's correlation coefficient analysis and a 95\% confidence interval. Reliability of right subclavian artery IMT measurement for the diagnosis of early thickening (considering a $>0.8 \mathrm{~mm}$ carotid thickness as reference) was described as to sensitivity, specificity, positive predictive value, negative predictive value, and accuracy. Cut-off values for the right subclavian IMT were indicated by the ROC curve, and $p$ values $\leq 0.05$ were considered statistically significant.

Results: Out of the 41 patients whose carotid arteries were IMT-free, 30 (73\%) had right subclavian artery IMT values $>0.8 \mathrm{~mm}$. The mean IMT value for the carotid artery was $0.87 \mathrm{~mm}(S D=0.23)$ and for the subclavian artery, $1.17 \mathrm{~mm}(S D=0.46)$, with a 0.31 correlation coefficient (95\% Cl: 0.12; 0.47). The ROC curve analysis indicated a cut-off value of $0.7 \mathrm{~mm}$ for the right subclavian artery IMT, using as reference a $0.8 \mathrm{~mm}$ cut-off value for the carotid artery (91\% sensitivity, 27\% specificity, 66\% PPV, 65\% NPV, and 66\% accuracy).

Conclusion: Our study showed that carotid artery IMT correlates well with right subclavian artery IMT. With a 0.7 mm cut-off value, it is possible to detect IMT in the right subclavian artery earlier than in the carotid arteries. The IMT at the origin of the right subclavian artery can be considered an earlier marker for the assessment of cardiovascular risk.

Key words: Media-intima thickening, coronary arteriosclerosis, myocardial infarction, early diagnosis. 
A aterosclerose é uma doença generalizada da parede arterial, que evolutivamente pode progredir, regredir ou estabilizar-se na dependência de uma série de fatores ${ }^{1}$. Esse processo dinâmico é caracterizado por remodelamento na parede arterial que pode permanecer silencioso por muito tempo ou pode manifestar-se como um evento vascular agudo, tornando-se clinicamente aparente. A detecção de marcadores para doença cardiovascular possibilita a intervenção precoce sobre os fatores de risco modificáveis para doença aterosclerótica, como alteração do estilo de vida, tratamento agressivo da hipertensão arterial, dislipidemia e diabete melito ${ }^{2}$

O espessamento médio-intimal (EMI) da artéria carótida, medido pela ultra-sonografia vascular de alta resolução, é considerado atualmente um marcador de doença aterosclerótica generalizada, principalmente da doença arterial coronariana precoce ${ }^{2-8}$. $\mathrm{O}$ aumento do complexo médio-intimal da artéria carótida está relacionado com a maioria dos fatores de risco cardiovasculares: sexo masculino, história familiar de acidente vascular cerebral ou infarto agudo do miocárdio, tabagismo, diabete melito, dislipidemia, hipertrofia do ventrículo esquerdo, hiper-homocisteinemia e idade $^{9-17}$. O EMI da artéria carótida ajuda a estabelecer com mais precisão o risco cardiovascular em pacientes hipertensos sem lesão em órgão-alvo evidenciada pelos exames de rotina, como o eletrocardiograma ${ }^{18-20}$.

O EMI, como marcador de risco cardiovascular, pode ser detectado na artéria carótida comum distal, na bifurcação carotídea, na carótida interna e, mais recentemente, na artéria femoral comum ${ }^{21,22}$. No entanto, estudos demonstraram que a medida do EMI na carótida interna é a que apresenta melhor correlação com os fatores de risco para doença arterial coronariana $^{23}$.

No estudo rotineiro do tronco braquiocefálico e da origem da artéria subclávia direita nos pacientes enviados ao nosso laboratório vascular para avaliação de risco cardiovascular pela mensuração do EMI nas artérias carótidas foi observado que alguns pacientes sem espessamento do complexo médiointimal nas artérias carótidas apresentavam EMI na origem da artéria subclávia direita, por isso o objetivo deste trabalho foi investigar a existência de associação entre o EMI nas artérias carótidas e na origem da artéria subclávia direita e avaliar se o EMI na artéria subclávia direita poderia ser considerado como um marcador mais precoce para doença cardiovascular.

\section{Métodos}

Cento e seis pacientes consecutivos foram submetidos a avaliação das artérias carótidas e subclávias direitas pela ultrasonografia vascular de alta resolução.

Os critérios de inclusão no estudo foram indivíduos assintomáticos com fator de risco para doença arterial coronariana. Tais fatores de risco incluíam: idade maior que 55 anos em homens e maior que 65 anos em mulheres, hipertensão arterial sistêmica, diabete melito, tabagismo, dislipidemia, obesidade, sedentarismo e história familiar de doença arterial coronariana precoce.

Os critérios de exclusão foram indivíduos sem fatores de risco para doença cardiovascular e a presença de placa aterosclerótica nas artérias carótidas evidenciada durante a ultra-sonografia vascular com Doppler colorido.

A medida do EMI foi realizada na carótida comum distal (1-2 cm proximal à bifurcação carotídea) e na carótida interna bilateralmente, assim como na origem da artéria subclávia direita. Em toda a análise foi considerado o maior valor de EMI nas artérias carótidas direita e esquerda e o valor mensurado na origem da artéria subclávia direita. A artéria subclávia direita pode ser avaliada com facilidade, por localizar-se mais superficialmente que a artéria subclávia contra-lateral; contudo, não apresenta vantagens ou limitações técnicas em relação às artérias carótidas. A artéria subclávia esquerda não foi incluída no estudo em razão de sua localização mais profunda que limita a avaliação da origem desse vaso.

A medida do complexo médio-intimal foi realizada com aparelho de ultra-som vascular Siemens Sonoline Elegra ${ }^{\circledR}$. Foi utilizado transdutor linear de $7,5 \mathrm{mHz}$, com freqüência entre 7 e $9 \mathrm{mHz}$, em seção longitudinal, no modo B. A medida do espessamento foi realizada na parede anterior ou posterior da artéria, na distância entre duas linhas ecogênicas representadas pelas interfaces lúmen-íntima e média-adventícia da parede arterial $^{17,24-26}$.

Para avaliar a associação entre EMI das artérias carótida e subclávia direita, calcularam-se o coeficiente de correlação de Pearson e o intervalo de 95\% de confiança para esse coeficiente. Considerando-se a classificação pela carótida como padrão de referência no diagnóstico de espessamento precoce (valores $\geq 0,8 \mathrm{~mm}$ indicaram espessamento precoce), ajustou-se uma curva ROC aos valores da EMI da subclávia e determinou-se o ponto de corte para essa mesma classificação. Para avaliar o resultado obtido, foram calculados os valores de sensibilidade, especificidade, valor preditivo positivo, valor preditivo negativo e acurácia para a EMI da subclávia na identificação do espessamento precoce. Para a avaliação do método estudado, dois observadores independentes mediram a EMI da carótida de treze pacientes para uma análise da reprodutibilidade. Essa análise foi feita por meio de componentes de variância. O nível de confiança de 95\% foi adotado em toda a análise.

\section{Resultados}

Foram avaliados 52 homens (49\%) e 54 mulheres (51\%), com idade entre 23 e 83 anos (média $51 \pm$ 13,19 anos).

A prevalência dos fatores de risco analisados para doença arterial coronariana está descrita na tabela 1.

Correlação entre EMI na artéria carótida e EMI na artéria subclávia direita - Considerando o critério de $>0,8 \mathrm{~mm}$ para EMI na artéria subclávia direita, 41 artérias carótidas não apresentaram EMI, e trinta (73\%) artérias subclávias direitas apresentavam EMI > 0,8 mm. Das 65 artérias carótidas com espessamento precoce, 59 (91\%) artérias subclávias apresentaram EMI $>0,8 \mathrm{~mm}$.

O valor médio de EMI obtido na artéria carótida (Gráfico 1) foi de 0,87 mm (mínimo 0,5 mm, máximo 1,3 mm, mediana $0,9 \mathrm{~mm}$ e desvio padrão 0,23 ). O valor médio de EMI obtido na artéria subclávia direita (Gráfico 2) foi de 1,17 mm (mínimo 0,4 mm, máximo 2,8 mm, mediana 1,1 mm e desvio padrão $0,46)$. A análise de reprodutibilidade do método indicou um 


\begin{tabular}{|lc|}
\hline Fator de Risco para DAC & Número de Pacientes \\
\hline Hipertensão Arterial Sistêmica & 36 \\
\hline Diabete melito & $34 \%$ \\
\hline Tabagismo & 4 \\
& $4 \%$ \\
Dislipidemia & 28 \\
& $26 \%$ \\
Obesidade & 39 \\
& $37 \%$ \\
Sedentarismo & 19 \\
& $18 \%$ \\
História familiar de DAC & 30 \\
\hline Tabela 1 - Prevalência dos fatores de risco para doença arterial & $28 \%$ \\
& coronariana (DAC) \\
\hline
\end{tabular}

erro percentual de 10,2\% entre os observadores.

O coeficiente de correlação entre o EMI da artéria carótida e da artéria subclávia direita foi de 0,31, com um intervalo de $95 \%$ de confiança de 0,12 a 0,47.

EMI da artéria subclávia como indicador de espessamento precoce - Determinado o espessamento da carótida com ponto de corte de $0,8 \mathrm{~mm}$ como padrão de referência para o diagnóstico de EMI precoce, os valores de EMI da carótida menores que $0,8 \mathrm{~mm}$ foram considerados normais e valores superiores a 0,8 $\mathrm{mm}$ considerados espessamento precoce. O ponto de corte, pela curva ROC (Gráfico 3), para o EMI na origem da artéria subclávia direita que melhor classifica $\mathrm{O}$ espessamento foi o valor de 0,7 $\mathrm{mm}$.
O ponto de corte de $0,7 \mathrm{~mm}$ na origem da artéria subclávia direita, comparado ao padrão de referência $(0,8$ $\mathrm{mm}$ ) do EMI nas artérias carótidas apresentou sensibilidade, especificidade, VPP, VPN e acurácia de 91\%, 27\%,66\%, 65\% e $66 \%$, respectivamente. Os valores de sensibilidade para outros pontos de corte do EMI na artéria subclávia direita podem ser observados na Tabela 2.

\section{Discussão}

A identificação de alterações na parede arterial de indivíduos assintomáticos indica um controle mais rigoroso dos fatores de risco cardiovasculares, com o objetivo de prevenir um evento coronariano futuro.

Estudos populacionais e hospitalares utilizaram técnicas não-invasivas para avaliar alterações precoces na estrutura e função da parede arterial, como a medida do complexo médio-intimal, pesquisa de disfunção endotelial e calcificação arterial coronariana ${ }^{25,27,28}$.

A medida do EMI da artéria carótida é um método de fácil reprodutibilidade, seguro e baixo custo, que identifica pacientes com doença aterosclerótica subclínica e com maior risco de desenvolver doença arterial coronariana ${ }^{7,25,29,30}$.

Estudos determinaram o valor de $0,8 \mathrm{~mm}$ para espessamento precoce do complexo médio-intimal, relacionado a aumento do risco cardiovascular ${ }^{4,18,31-33}$. Groot e cols. realizaram um estudo com 315 pacientes com hipercolesterolemia familiar comparados com 118 controles, e mostraram que um espessamento do complexo médio-intimal de até 0,8 $\mathrm{mm}$ seria considerado normal. Os pacientes do grupo com hipercolesterolemia familiar atingiram o valor de $0,8 \mathrm{~mm}$ de EMI aos quarenta anos; já os pacientes do grupo controle atingiam esse valor apenas aos 76 anos, quando seu risco cardiovascular aumentaria por causa da idade ${ }^{4}$.

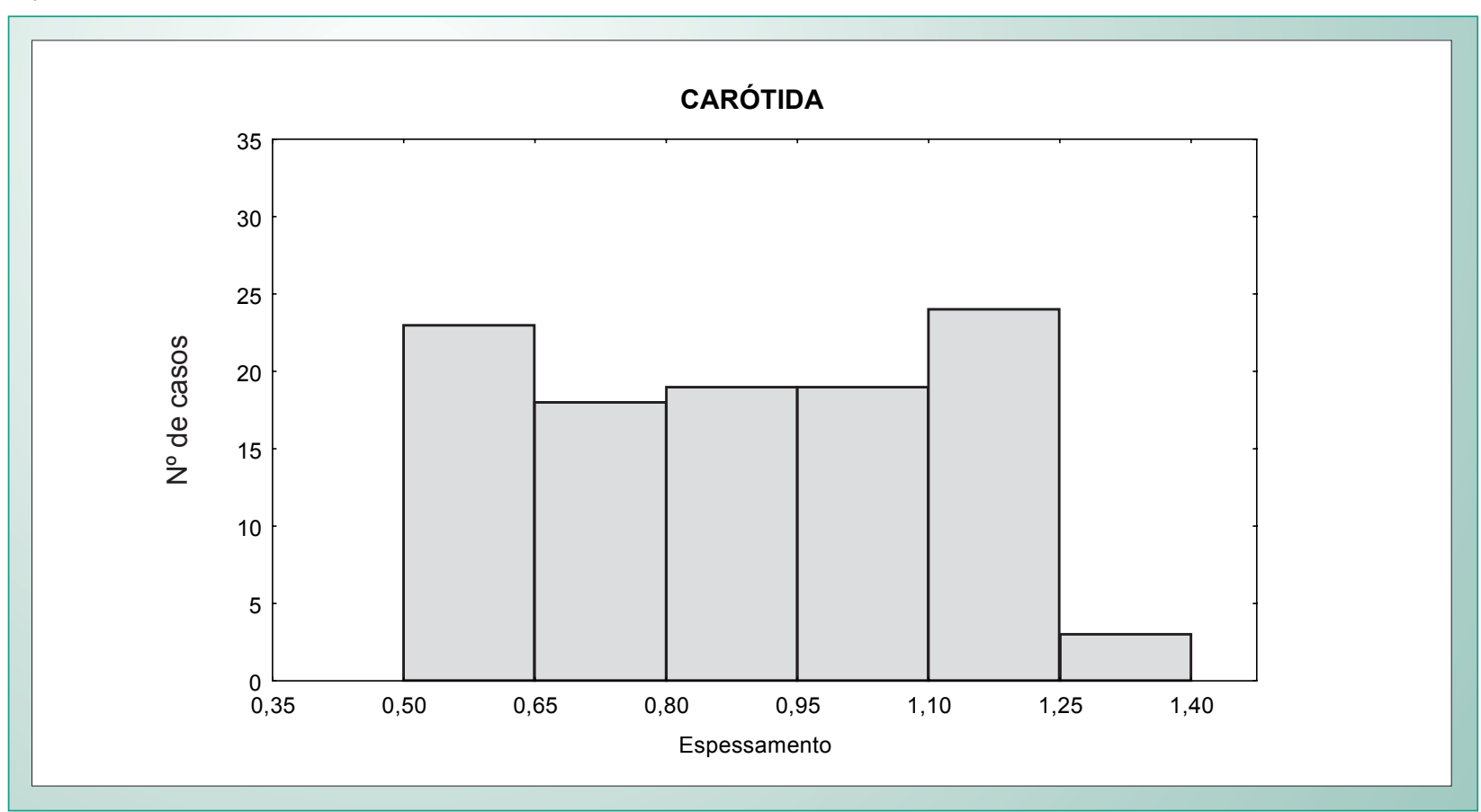

Gráfico 1 - Distribuição dos maiores valores de EMI observados nas artérias carótidas. 


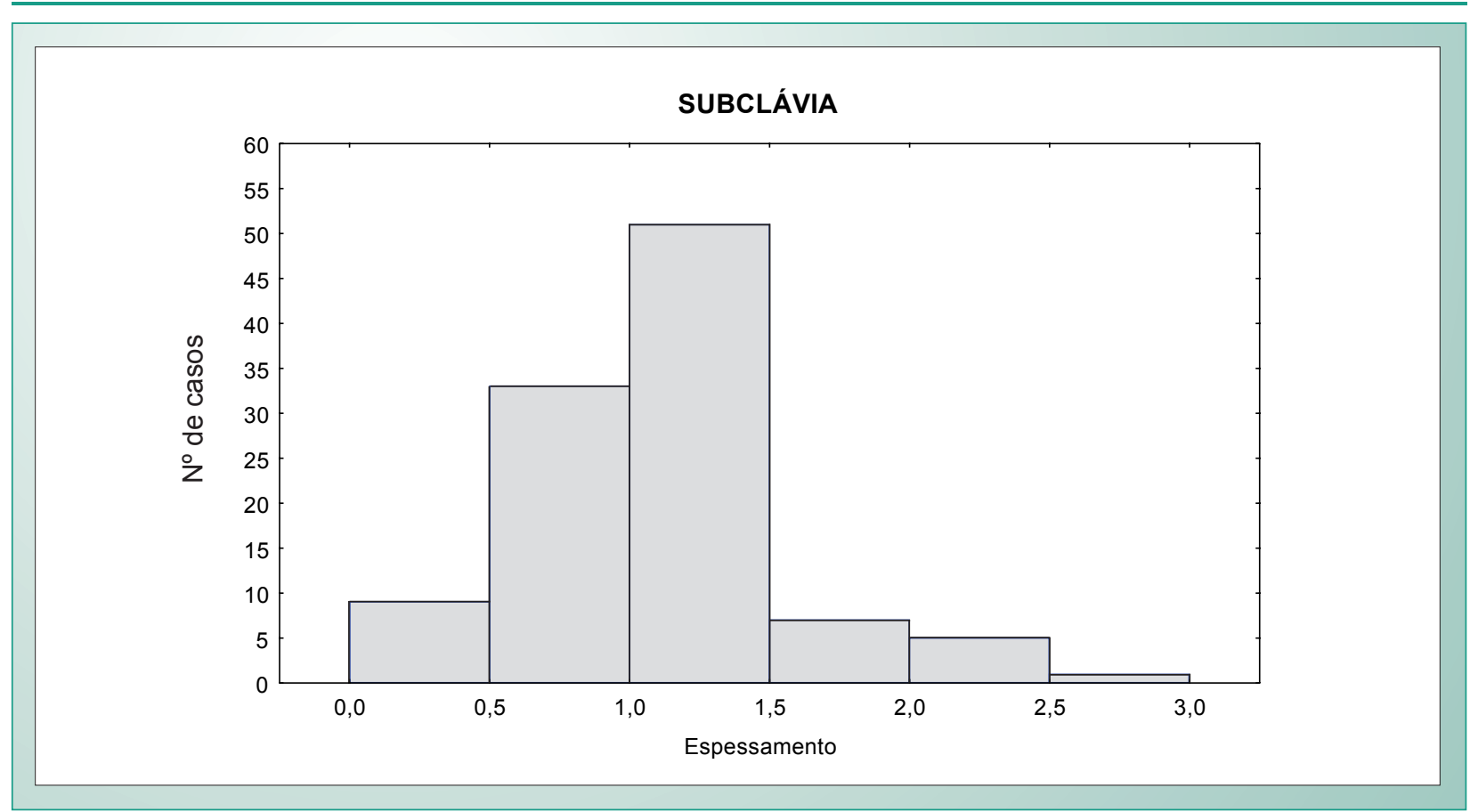

Gráfico 2 - Distribuição dos valores de EMI observados na artéria subclávia direita.

\begin{tabular}{|c|c|c|c|c|c|}
\hline Índices & $<0,8 ; \geq 0,8$ & $<0,9 ; \geq 0,9$ & $<1 ; \geq 1$ & $<1,1 ; \geq 1,1$ & $<1,2 ; \geq 1,2$ \\
\hline Sensibilidade & $90,8 \%$ & $81,5 \%$ & $81,5 \%$ & $67,7 \%$ & $56,9 \%$ \\
\hline Especificidade & $26,8 \%$ & $31,7 \%$ & $36,6 \%$ & $51,2 \%$ & $75,6 \%$ \\
\hline $\mathrm{VP}+$ & $66,3 \%$ & $65,4 \%$ & $67,1 \%$ & $68,8 \%$ & $78,7 \%$ \\
\hline VP- & $64,7 \%$ & $52,0 \%$ & $55,6 \%$ & $50,0 \%$ & $52,5 \%$ \\
\hline Eficiência & $66,0 \%$ & $62,3 \%$ & $64,2 \%$ & $61,3 \%$ & $64,2 \%$ \\
\hline
\end{tabular}

Tabela 2 - Índices de qualidade do espessamento da artéria subclávia direita considerando-se diferentes pontos de corte

Indivíduos assintomáticos, com probabilidade pré-teste baixa a intermediária para a presença de doença arterial coronariana, apresentam maior risco de eventos coronarianos futuros caso apresentem EMI da carótida maior que 1 $\mathbf{m m}^{8,25,29,30,34}$. Estudos multicêntricos demonstraram que pacientes com EMI superior a $1 \mathrm{~mm}$ apresentam risco maior de infarto agudo do miocárdio em quatro anos , 29,30 $^{\text {C Como o }}$ objetivo do nosso estudo foi determinar um marcador mais precoce de risco cardiovascular, utilizamos o ponto de corte de $0,8 \mathrm{~mm}$.

O coeficiente de correlação entre o EMI da artéria carótida e da artéria subclávia direita, neste estudo, foi de 0,31, com um intervalo de 95\% de confiança de 0,12 a 0,47. O fato do zero não estar incluído nesse intervalo indica a significância dessa correlação; entretanto, observando-se os limites de 95\% de confiança, percebe-se que apesar do baixo limite inferior, o limite superior indica uma boa correlação entre o EMI da artéria carótida e da artéria subclávia direita.

Ainda nesse estudo foi determinado pela sensibilidade de $91 \%$ o valor do EMI na artéria subclávia direita $(0,7 \mathrm{~mm})$ inferior ao valor referencial para o EMI da artéria carótida $(0,8$ $\mathrm{mm})$, o que sugere que a medida do espessamento da origem da artéria subclávia possa ser um marcador mais precoce de risco cardiovascular.

O EMI, medido na imagem em modo B do ultra-som, consiste na distância entre duas linhas ecogênicas representadas pelas interfaces lúmen-íntima e média-adventícia da parede arterial. A ultra-sonografia vascular, mesmo de alta resolução, é incapaz de diferenciar a camada íntima da camada média da parede arterial; portanto, rotineiramente o complexo médiointimal é mensurado. Um aumento na espessura do complexo médio-intimal pode ser devido tanto a espessamento da camada média como da camada íntima. Sabe-se que a doença aterosclerótica afeta predominantemente a camada íntima da parede arterial. As artérias carótidas e subclávias são artérias elásticas, com um componente muscular muito pequeno e predomínio da camada íntima. Em contraste, nas artérias periféricas como a artéria femoral, observa-se predomínio da camada muscular média. Portanto, o EMI nas artérias carótidas e subclávias representa principalmente 


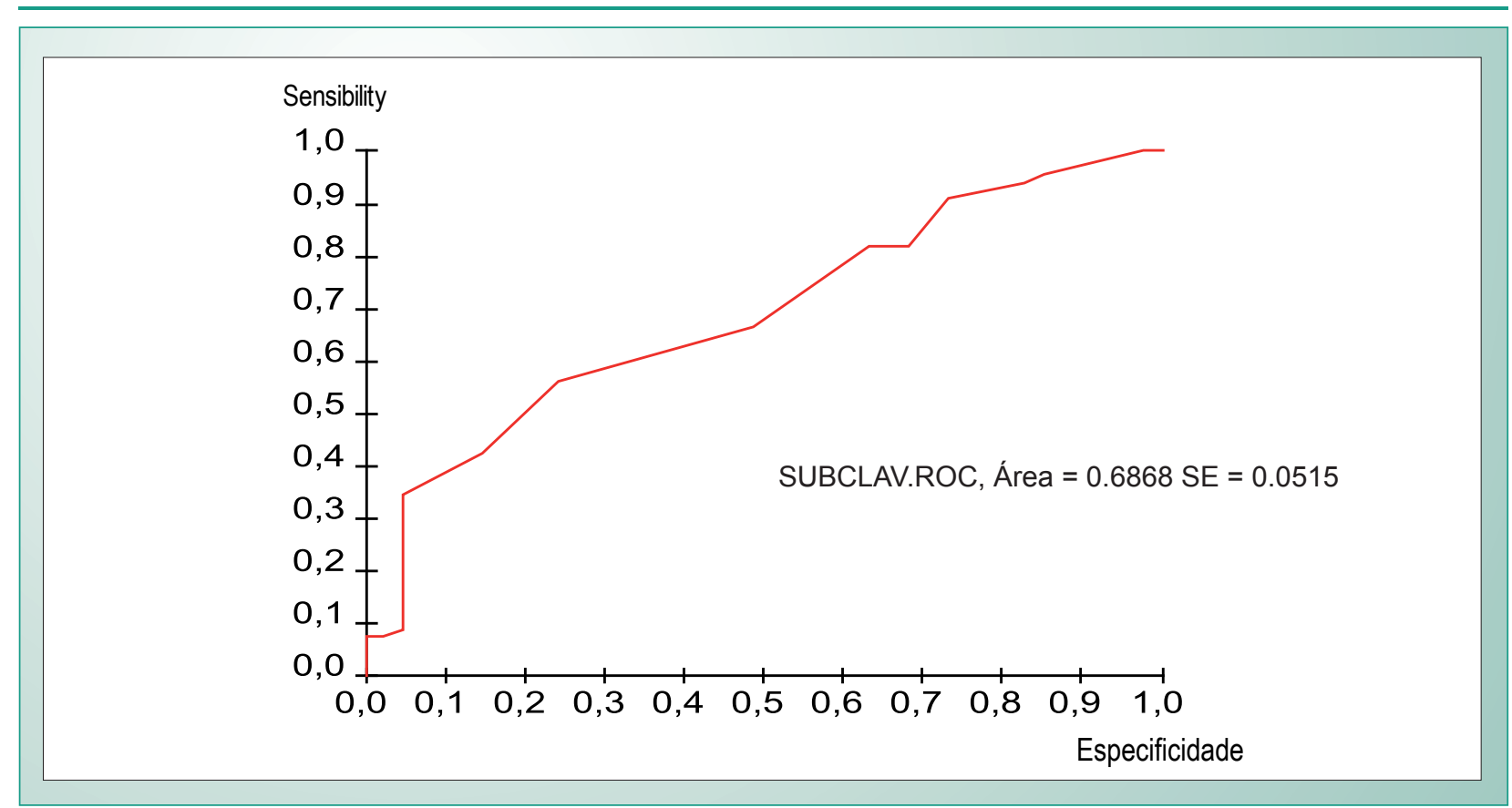

Gráfico 3 - Dados da Curva ROC para definição do ponto de corte para o EMI da artéria subclávia direita que melhor classifica o espessamento.

o espessamento da camada íntima, o qual está relacionado com a presença de doença aterosclerótica ${ }^{3,26,35,36}$. O EMI da artéria carótida superior a 1,3 mm já é considerado placa aterosclerótica $^{37}$.

Uma possível explicação para o aparecimento mais precoce de espessamento médio-intimal na artéria subclávia direita seria a presença de maior angulação do vaso observada na origem dessa artéria em relação à bifurcação carotídea. A maior velocidade existente junto à borda interna das curvaturas, como observada na origem da artéria subclávia direita, é responsável por aumentar o estresse da superfície endotelial e das forças de cisalhamento aí existentes. ${ }^{38} \mathrm{O}$ estresse na parede arterial e a conseqüente maior força de cisalhamento contribuiria para o desenvolvimento de espessamento médio-intimal e posteriormente da placa aterosclerótica no local. Já em 1963, Texon formulou a hipótese de que a menor pressão no lado interno favoreceria o aparecimento de deposições ateromatosas nas curvaturas ${ }^{39}$. Contudo, não foram identificados estudos na literatura relacionando EMI da artéria subclávia direita com fatores de risco de doença cardiovascular.

\section{Referências}

1. Badimon JJ, Fuster V, Chesebro JH, Badimon L. Coronary atherosclerosis: a multifactorial disease. Circulation 1993; 87 (suppl): II3-II16.

2. Wittes J, Lakatos J, Probstfield J. Surrogate endpoints in clinical trials. Stat Med 1989; 8: 415-25.

3. Grobbee DE, Bots ML. Carotid artery intima-media thickness as an indicator of generalized atheroclerosis. J Int Med 1994; 236: 567-73.

4. Groot E, Hovingh GK, Wiegman A, et al. Measurement of arterial wal thickness as a surrogate marker for atherosclerosis. Circulation 2004; 109 (suppl III): III-33-III-38.
Esse trabalho observou que em 41 pacientes com artérias carótidas sem EMI, trinta (73\%) artérias subclávias direitas apresentavam EMI $>0,8 \mathrm{~mm}$. Esse achado demonstra que, mesmo quando a artéria carótida estiver normal, pode-se encontrar espessamento do complexo médio-intimal na artéria subclávia; portanto, a presença de EMI pode ser detectada mais cedo na artéria subclávia do que na artéria carótida, permitindo indicar mais precocemente o controle agressivo dos fatores de risco cardiovasculares a fim de prevenir a ocorrência de um evento coronariano futuro.

Os autores concluem que existe associação entre o EMI das artérias carótidas e na origem da artéria subclávia direita. O EMI pode ser detectado mais precocemente na artéria subclávia do que nas artérias carótidas, com valor de corte de 0,7 mm. O EMI na origem da artéria subclávia direita pode ser considerado um marcador mais precoce para avaliação de risco cardiovascular.

\section{Potencial Conflito de Interesses}

Declaro não haver conflitos de interesses pertinentes.

5. Van Bortel LM. What does intima-media thickness tell us? J Hypertens 2005; 23: 37-9.

6. Bots ML, Grobbee DE, Hofman A, Witteman JCM. Common carotid intimamedia thickness and risk of acute myocardial infaction. Stroke 2005; 36 : 762-7.

7. O'Leary DH, Polak JF, Kronmal RA, Manolio TA, Burke GL, Wolfson SK Jr. Carotid-artery intima and media thickness as a risk factor for myocardial infarction and stroke in older adults. Cardiovascular Health Study Collaborative Research Group. N Engl J Med 1999; 340: 14-22. 


\section{Artigo Original}

8. Simon A, Gariepy J, Chironi G, Mengnien JL, Levenson J. Intima-media thickness: a new tool for diagnosis and treatment of cardiovascular risk. J Hypertens 2002; 20: 159-69.

9. Crouse JR, Tang R, Espeland MA, Terry JG, Morgan T, Mercuri M. Association of extra-cranial carotid atheroclerosis progression with coronary status and risk factors in patients with and without coronary artery disease. Circulation 2002; 106: 2061-6.

10. Luedemann J, Schminke U, Berger K, et al. Association between behaviordependent cardiovascular risk factors and asyntomatic carotid atheroclerosis in a general population. Stroke 2002; 33: 2929-35.

11. Stein JH, Douglas PS, Srinivasan SR, et al. Distribution and cross-sectional age-related increases of carotid artery intima-media thickness in young adults. The Bogalusa Heart Study. Stroke 2004; 35: 2782-7.

12. Jerrard-Dunne P, Markus HS, Steckel Da, Buehler A, von Kegler S, Stizer M. Early carotid atherosclerosis and family history of vascular disease. Specific effects on arterial sites have implications for genetic studies. Arterioscler Thromb Vasc Biol 2003; 23: 302-6.

13. Weber F. Risk factors for subclinical carotid atherosclerosis in healthy men Neurology 2002; 59: 524-28.

14. Tropeano AI, Boutouyrie P, Katsahian S, Laloux B, Laurent S. Glucose level is a major determinant of carotid intima-media thickness in patients with hypertension and hyperglycemia. J Hipertens 2004; 22: 2153-60.

15. Liu ML, Yilitalo K, Salonen R, Salonen JT, Taskinen MR. Circulating oxidized low-density lipoprotein and its association with carotid intima-media thickness in asyntomatic members of familial combined hiperlipidemia families. Arterioscler Thromb Vasc Biol 2004; 24: 1492-7.

16. Wang TJ, Nam BH, D'Agostinho RB, et al. Carotid intima-media thickness is associated with premature parental coronary heart disease. The Framinghan Heart Study. Circulation 2003; 108: 572-6.

17. Vaudo G, Schillaci G, Evangelista F, Pasqualini L, Verdecchia P, Mannarino E. Arterial wall thickening at different sites and its association with left ventricular hypertrophy in newly diagnosed essential hypertension. Am J Hypertens 2000; $13: 324-31$.

18. Cuspidi C, Ambrosioni E, Mancia G, Pessina AC, Trimarco B, Znachetti A. Role o echocardiography and carotid ultrasonography in stratifying risk in patients with essential hypertension: the Assesment of Prognostic Risk Observational Survey. J Hypertens 2002; 20: 1307-14

19. Zakopoulos NA, Tsivgoulis G, Barlas G, et al. Time rate of bood pressure variation is associated with increased common carotid artery intima-media thickness. Hypertension 2005; 45: 505-12.

20. Guidelines committee. 2003 European Society of Hypertension - European Society of Cardiology guidelines for manegement of arterial hypertension. J Hypertens 2003; 21: 1011-53.

21. Held C, Hjemdahl P, Eriksson SV, Björkander I, Forslund L, Rehnquivist N. Prognostic implications of intima-media thickness and plaques in the carotid and femoral arteries in patiens with stable angina. Eur Heart J 2001; 22: 6272

22. Lelakis JP, Papamichael CM, Cimponeriu AT, et al. Atherosclerotic changes of extra-coronary arteries are associated with the extent of coronary atheroclerosis. Am J Cardiol 2000; 85: 949-52.

23. Mackinnon AD, Jerrard-Dunne P, Tizer M, Buehler A, von Kegler S, Markus HS. Rates and determinants of site-specific progression of carotid artery intima-media thickness. The carotid atheroclerosis progression study. Stroke
2004; $35: 2150-4$.

24. Pignoli P, Tremoli E, Poli A, Oreste P, Paoletti R. Intimal plus medial thickness of the arterial wall: a directed mesurement with ultrasound imaging. Circulation 1986; 74: 1399-406.

25. Bots ML, Dijk JM, Oren A, Grobbee DE. Carotid intima-media thickness, arterial wall stiffness and risk of cardiovascular disease: current evidence. J Hipertens 2002; 2317-25.

26. Sinha AK, Eigenbrodt M, Mehta JL. Does carotid intima media thickness indicate coronary atheroclerosis? Curr Opin Cardiol 2002; 17: 52630 .

27. Simon A, Megnien JL, Levenson J. Coronary risk estimation and treatment of hypercholesterolemia. Circulation 1997; 96: 2449-52.

28. Hollander M, Hak AE, Koudstaal PJ, et al. Comparison between measures of atheroclerosis and risk of stroke. Stroke 2003; 34: 2367 73.

29. Chambless LE, Heiss G, Folson AR, Szklo M, Sharrett AR, Cleg LX. Association of coronary heart disease incidence with carotid arterial wall thickness and major risk factors: The Atherosclerosis Risk in Communities (ARIC) Study. Am J Epidemiol 1997; 146: 483-94.

30. Bots ML, Hoes AW, Koudstaal PJ, Hofman A, Grobbee DE. Common carotid intima-media thickness and risk of stroke and miocardial infaction: the Rotterdan Study. Circulation 1997; 96:1432-7.

31. Jadkav UM, Kaddam NN. Carotid intima-media thickness as an independent predictor of coronary artery disease. Indian Heart J 2001; 53 (4): 458-62

32. Rohani $\mathrm{M}$, Jogestrand $\mathrm{T}$, Ekberg $\mathrm{M}$, et al. Interrelation between the extent of atherosclerosis in the thoracic aorta, carotid intima-media thickness and the extent of coronary artery disease. Atherosclerosis 2005; 179 (2): 311-6.

33. Hodis HN, Mack WJ, Selzer RH, Liu C, Azen SP. The role of carotid arterial intima-media thickness in predicting clinical coronary events. Ann Intern Med 1998; 128: 262-9.

34. Aminbakhsh A, Mancini GB. Carotid intima-media thickness mesurements: waht defines an abnormality? A systematic review. Clin Invest Med 1999; 4: 149-57.

35. Slonen JT, Salonen R. Ultrasound B-mode imaging in observational studies of atherosclerotic progression. Circulation 1997; 87 (3 Suppl): II56-65.

36. Montenegro MRG. Estrutura da parede vascular. In Maffei FHA, Lastória S, Yoshida WB, Rollo HA. (ed.). Doenças Vasculares Periféricas. 3 ed. Rio de Janeiro: Medsi; 2002, v.1, 179-91.

37. Zanchetti A, Bond MJ, Henning M, et al. On behalf of the ELSA investigators. Risk factors associated with alterations in carotid intimamedia thickness in hypertension: baseline data from the European Lacidipine Studty on Atherosclerosis. J Hyperten 1998; 16: 949-61.

38. Lopes OU. Estudo das doenças vasculares periféricas a partir da dinâmica dos fluidos. In: Maffei FHA, Lastória S, Yoshida WB, Rollo HA. (ed.). Doenças vasculares periféricas. 3 ed., Rio de Janeiro: Medsi; 2002, v.1, 217-31.

39. Texon M. The role of vascular dynamics in the development of atherosclerosis. In Sandler M, Bourne GH (ed.). Atherosclerosis and Its Origiti. New York: Academic Press, 1963: 167-95. 